\title{
The forgotten cause of hypertension: A case report and literature review of the prevalence, diagnosis and management of primary aldosteronism
}

\author{
Mariah Pagath Barlow*1, Ahmed Abdel-Latif ${ }^{2}$ \\ ${ }^{1}$ Department of Internal Medicine, University of Kentucky Medical Center, Lexington, United States of America \\ ${ }^{2}$ Department of Cardiology, Gill Heart Institute, University of Kentucky, Lexington, United States of America
}

Received: January 19, 2018

DOI: $10.5430 /$ crim.v5n2p4

\author{
Accepted: March 14, 2018 \\ Online Published: March 28, 2018 \\ URL: https://doi.org/10.5430/crim.v5n2p4
}

\begin{abstract}
In recent years, primary aldosteronism (PA) has been found to have a much higher prevalence than previously thought, especially among those with resistant hypertension. As hypertension has become a prominent public health issue affecting billions of people, the number of patients potentially affected by PA is a significant. Current medical and surgical therapies for PA are highly effective, and as untreated PA can lead to significant cardiovascular morbidity and mortality this stresses the importance of astute diagnosis and management on part of physicians. We present a case of a patient presenting with cardiovascular complications of untreated primary aldosteronism and review recent guidelines regarding screening, diagnosis, and management.
\end{abstract}

Key Words: Conn's Syndrome, Adrenal hyperplasia, Primary aldosteronism, Hyperaldosteronism, Resistant hypertension

\section{BACKGROUND}

Primary aldosteronism (PA), or more commonly known as primary hyperaldosteronism, is the leading cause of secondary hypertension. ${ }^{[1,2]}$ Once thought to be an uncommon disease, recent studies have shown that PA is increasingly common and present in anywhere from $5 \%$ to $10 \%$ of all those with hypertension. As hypertension is one of the most common chronic medical conditions, it is imperative that physicians involved in long-term management of these patients are familiar with recent guidelines surrounding PA diagnosis and management. When left undiagnosed, patients with primary aldosteronism have increased risk of cerebrovascular and cardiovascular events, which stresses the importance of proper screening, especially as current medical and surgical therapies are highly successful. We report a case of primary aldosteronism in a patient presenting with a cardiovascular event. Our case highlights the adverse complications of long standing primary aldosteronism and reviews the current literature regarding the prevalence of PA as well as its diagnosis and long-term management.

\section{Case history}

A 65-year-old male with past medical history of poorly controlled hypertension presented to emergency department with acute onset substernal chest pain. His only known medical history was that of long-standing poorly controlled hypertension, that was self reported by the patient and confirmed by review of his previous blood pressure recordings and outpatient provider notes over the past several years in the hospital's electronic medical record. He was a lifelong non-

\footnotetext{
*Correspondence: Mariah Pagath Barlow; Email: mpa255@uky.edu; Address: 900 South Limestone St., Wethington Bldg 304B, Lexington, KY, United States of America.
} 
smoker and was non-obese with a BMI of 25. He had no history of hyperlipidemia. He did have a family history of coronary artery disease in his biological sister. On initial evaluation in the emergency department, he was hypertensive with a systolic blood pressures greater $>170 \mathrm{mmHg}$. His initial EKG was consistent with left ventricular hypertrophy, but no acute changes suggestive of underlying myocardial ischemia were seen. Initial troponin I was elevated at $0.056 \mathrm{ng} / \mathrm{dl}$ and serial troponins obtained following presentation demonstrated a significant delta change over the following 12 hours consistent with a non-STE myocardial infarction. He was loaded on dual anti-platelet therapy with aspirin $325 \mathrm{mg}$ and clopidogrel $600 \mathrm{mg}$ and started on a therapeutic unfractionated heparin drip. He was also started on high intensity statin therapy with atorvastatin $80 \mathrm{mg}$ daily. Beta blocker therapy was not administered as his heart rate was continuously 50 to 60 beats per minute. He was taken for coronary angiography the morning follow his presentation. Coronary angiogram demonstrated $80 \%$ ostial stenosis of the left circumflex artery for which percutaneous coronary intervention was performed with placement of two overlapping drug eluting stents. Echocardiogram obtained after the intervention demonstrated severe left ventricular hypertrophy and grade III diastolic dysfunction. Following the procedure, he remained hypertensive, despite up-titration to maximal doses of nifedipine, lisinopril and hydrochlorothiazide. He was also persistently hypokalemic with potassium levels ranging from 2.5 to $3.4 \mathrm{mmol} / \mathrm{L}$, necessitating aggressive oral potassium supplementation. Given concern for secondary hypertension, plasma aldosterone and renin levels were drawn and were $17.5 \mathrm{ng} / \mathrm{dl}$ and $0.466 \mathrm{ng} / \mathrm{ml} / \mathrm{hr}$ respectively, giving an elevated aldosterone renin ratio (ARR) of 37.5. A non-enhanced CT of the abdomen was performed and demonstrated slight enlargement and irregular thickening of the left adrenal gland. The hounsfield units of the area of enlargement of the left adrenal gland were low at 0 units, with $\mathrm{HU}<10$ being highly specific and diagnostic of a lipid-laden adrenal adenoma. ${ }^{[3]}$ Given the concern for a functioning adrenal adenoma, the patient underwent confirmatory testing with adrenal vein sampling. Serum aldosterone level from the left adrenal vein was significantly elevated level at $4,489 \mu \mathrm{g} / \mathrm{dl}$, while the right adrenal vein demonstrated normal secretion with a value of $16 \mu \mathrm{g} / \mathrm{dl}$. He was diagnosed with primary aldosteronism secondary to a unilateral adrenal adenoma. Given his recent angioplasty and myocardial infarction, decision was made continue the patient on medical therapy with spironolactone for the first year following his myocardial infarction, with plan to perform a unilateral adrenalectomy when he was able to safely hold his anti-platelet agents and undergo surgery. He currently continues to take spironolactone $100 \mathrm{mg}$ daily, in addition to his other anti-hypertensives, and his blood pressure is well controlled $<140 / 90 \mathrm{mmHg}$.

\section{Discussion}

Primary aldosteronism is a disorder characterized by inappropriately elevated aldosterone secretion and was first described by Jerome W. Conn in 1955. ${ }^{[4]}$ The previous assumption that PA was a rare disease representing only a miniscule number of patients, has been overturned by evidence from multiple retrospective and prospective studies. Recent studies have demonstrated an overall prevalence of $5 \%$ to $10 \%$ among all hypertensive patients ${ }^{[5-11]}$ and as high as $20 \%$ among the population of patients considered to have resistant hypertension. ${ }^{[12]}$ When considering that around one-third of adults in the United States over the age of 18 have essential hypertension, ${ }^{[13]}$ the number of patients potentially with undiagnosed PA is staggering.

As seen in our patient, long standing primary aldosteronism and subsequent hypertension can lead to cardiovascular complications. When compared to age and gender matched essential hypertension patients with the same degree of blood pressure elevation, patients with PA have increased risk of stroke, non-fatal myocardial infarction, and atrial fibrillation. ${ }^{[14,15]}$ Our patient demonstrates a prime example of the cardiovascular effects that uncontrolled hypertension leads too witnessed by his NSTEMI presentation, along with the severe concentric left ventricular hypertrophy and grade 3 diastolic dysfunction found on his echocardiogram. Primary aldosteronism is also associated with abnormal glucose metabolism and insulin resistance, ${ }^{[16]}$ and treatment of PA with unilateral adrenalectomy has been shown to be protective against development of new onset diabetes mellitus. ${ }^{[17]}$

In May 2016, the Endocrine Society and several other national groups, including the American Heart Association, published a revised clinical guideline surrounding primary aldosteronism in the Journal of Clinical Endocrinology and Metabolism advocating for more aggressive screening guidelines for primary aldosteronism. ${ }^{[18]}$ The following paragraphs summarize in brief the 2016 guideline, highlighting what is most applicable for general practitioners.

Evaluation for PA is recommended in patients with any of the following: I) sustained hypertension $>150 / 100$ on three different days, II) persistent hypertension (> 140/90 $\mathrm{mmHg}$ ) in patients on three anti-hypertensives including 1 diuretic, III) controlled blood pressure $(<140 / 90 \mathrm{mmHg}$ ) on 4 or more anti-hypertensives, IV) hypertension with spontaneous or diuretic induced hypokalemia, V) hypertension and incidental adrenal mass discovered on abdominal scan, VI) hypertension and sleep apnea, VII) hypertension and family history 
of hypertension or cerebrovascular accident under the age of 40, and VIII) all hypertensive patients with family history of PA.

The plasma ARR is the initial screening test of choice. There is currently much discussion about the upper limit of normal for the ARR secondary to the variability of the varying laboratory assays. The overall range of what is considered an elevated ARR ranges from 20 to 40; the Endocrine Society reports a value $>30$ as the most commonly used cutoff. ${ }^{[18]}$ Given the variability in plasma renin levels which could be abnormally low in other processes besides primary aldosteronism, some experts recommend combining both an ARR > 30 along with a plasma aldosterone concentration $(\mathrm{PAC})>15$ in order to increase specificity of plasma testing. ${ }^{[18]}$ Decision not to screen based solely on the absence of hypokalemia should be avoided, as less then $50 \%$ of patient with primary aldosteronism are hypokalemic. ${ }^{[5,19]}$

As multiple anti-hypertensives (beta blockers, ace inhibitors, angiotensin reception blockers, clonidine, potassium sparing and wasting diuretics) can affect plasma aldosterone and renin levels, the recommendation is to hold these medications, if feasible, for at least two weeks prior to testing. In the event that affecting medications cannot be temporarily held, as often the case in patients with severe hypertension, testing can still be performed as long as results are interpreted while accounting for the medications' effect on the PAC and plasma renin concentration (PRC).

In patient with an elevated ARR, confirmatory testing is recommended before proceeding with adrenal imaging or adrenal vein sampling. However, in patients with an elevated ARR and either hypokalemia, a PAC $>20$ or undetectable PRC confirmatory, testing is not indicated. Current confirmatory tests available include captopril challenge test, fludrocortisone suppression, oral sodium loading, and saline infusion testing. ${ }^{[20]}$ If confirmatory testing is also positive, adrenal imaging is the next step in order to evaluate for large masses concerning for adrenocortical carcinoma and to help with procedural planning for interventional radiologists and general surgeons. CT is the preferred imaging modality as it is more cost-effective, and MRI has not been shown to offer advantages over CT.
If CT imaging is concerning of a unilateral adenaoma or hyperplasia, adrenal vein sampling is currently recommended as the next step prior to adrenalectomy to both confirm unilateral secretion and to rule out non-functioning adenomas at the cause of abnormal imaging findings. While this is the current recommendation, newer studies have demonstrated that in the future adrenal vein sampling may ultimately end up being unneeded. A recent study comparing 200 patients with primary aldosteronism who underwent adrenalectomy guided by CT guided images alone vs adrenal vein sampling showed no-significant difference in treatment outcomes of primary aldosteronism between the two groups highlighting that adrenalectomy guided by CT images only is likely a viable option. ${ }^{[21]}$ If a patient is unwilling to undergo surgery or is not a candidate for surgery, adrenal vein sampling is unnecessary and the patient should be managed with a mineralocorticoid receptor antagonist (MRA) such as spironolactone. For those patients who undergo adrenal vein sampling, if findings are consistent with unilateral secretion, they should undergo unilateral adrenalectomy. On the other hand, those with bilateral secretion are managed medically with MRAs.

In summary, primary aldosteronism has larger prevalence than previously assumed, and given the epidemic of hypertension both within the United State and worldwide, likely affects a significant number of patients. Early diagnosis is $\mathrm{key}$, as PA is an extremely treatable cause of reversible hypertension. When left untreated, PA is associated with an overall higher rate of cardiovascular morbidity and mortality. Going forward, cardiologists, endocrinologists and primary care physicians need to continue to work together to identify patients at risk of PA and ensure these patients are evaluated and managed appropriately.

\section{ACKNOWLEDGEMENTS}

Dr. Abdel-Latif is supported by the University of Kentucky Clinical and Translational Science Pilot Award (UL1TR000117), the UK COBRE Early Career Program (P20 GM103527) and the NIH Grant R56 HL124266.

\section{CONFlicts of InTERest Disclosure}

The authors declare that they have no competing interests.

\section{REFERENCES}

[1] Schwartz GL, Turner ST. Screening for primary aldosteronism in essential hypertension: diagnostic accuracy of the ratio of plasma aldosterone concentration to plasma renin activity. Clin Chem. 2005; 51 : 386-394. https://doi.org/10.1373/clinchem. 2004.041780
[2] Young WF. Primary aldosteronism: renaissance of a syndrome. Clin Endocrinol (Oxf). 2007; 66: 607-618. https://doi .org/10.111 $1 / j .1365-2265.2007 .02775 . x$

[3] Schieda N, Siegelman E. Update on CT and MRI of adrenal nodules. AJR. 2017 Jun; 208: 1206-1217. PMid:28225653. https: 
//doi.org/10.2214/AJR.16.17758

[4] Conn JW. Presidential address 1. painting the background II. primary aldosteronism a new clinical syndrome. J Lab Clin Med. 1955 Jan; 45(1): 3-17. PMid:13233623

[5] Rossi GP, Bernini G, Caliumi C, et al. A prospective study of the prevalence of primary aldosteronism in 1,125 hypertensive patients. J Am Coll Cardiol. 2006 Dec; 48(11): 2293-2300. https: //doi.org/10.1016/j.jacc.2006.07.059

[6] Fardella CE, Mosso L, Gomez-Sanchez C, et al. Primary hyperaldosteronism in essential hypertensives: prevalence, biochemical profile and molecular biology. J Clin Endocrinol Metab. 2000 May; 85(5): 1863-1870. https://doi.org/10.1210/jcem.85.5.6596

[7] Douma S, Petidis K, Doumas M, et al. Prevalence of primary hyperaldosteronism in resistant hypertension: a retrospective observational study. Lancet. 2008 Jun 7; 371(9628): 1921-1926. https: //doi.org/10.1016/S0140-6736(08)60834-X

[8] Monticone S, Burrello J, Tizzani D, et al. Prevalence and clinical manifestations of primary aldosteronism encountered in primary care practice. J Am Coll Cardiol. 2017 Apr 11; 69(14): 1811-1820. https://doi.org/10.1016/j.jacc.2017.01.052

[9] Gordon RD, Stowasser M, Tunny TJ, et al. High incidence of primary aldosteronism in 199 patients referred with hypertension. Clin Exp Pharmacol Physiol. 1994 Apr; 21(4): 315-318. PMid:7923898. https://doi.org/10.1111/j.1440-1681.1994.tb02519.x

[10] Galati SJ, Cheeseman KC, Springer-Miller R, et al. Prevelence of primary aldosteronism in an urban hypertensive population. Endocr Pract. 2016 Nov; 22(11): 1296-1302. https ://doi.org/10.415 8/E161332.OR

[11] Hannemann A, Bidlingmaier M, Friedrich N, et al. Screening for primary aldosteronism in hypertensive subjects: results from two German epidemiological studies. Eur J Endocrinol. 2012 Jul; 167(1): 7-15. https : //doi .org/10.1530/EJE-11-1013

[12] Mosso L, Carvajal C, Gonzalez A, et al. Primary aldosteronism and hypertensive disease. Hypertension. 2003 Aug; 42(2): 161-5. https://doi.org/10.1161/01.HYP.0000079505.25750.11

[13] Yoon SS, Fryar C, Carrol M. Hypertension prevalence and control among adults: United States, 2011-2014. National Center for Health Statistics data brief. 2015 Nov; (220): 1-8. https: //doi.org/10.1016/j.jacc.2005.01.015

[14] Milliez P, Girerd X, Plouin PF, et al. Evidence for an increased rate of cardiovascular events in patients with primary aldosteronism. $\mathrm{J}$ Am Coll Cardiol. 2005 Apr 19; 45(8): 1243-1248. PMid:15837256. https://doi.org/10.1016/j.jacc. 2005.01.015

[15] Abad-Cardiel M, Alvarez-Alvarez B, Luque-Fernandez L, et al. Hypertension caused by primary hyperaldosteronism: increased heart damage and cardiovascular risk. Rev Esp Cardiol (Engl Ed). 2013 Jan; 66(1): 47-52. https ://doi .org/10.1016/j.recesp. 2012. 07.025

[16] Chen W, Li F, He C, et al. Elevated prevalence of abnormal glucose metabolism in patients with primary aldosteronism: a meta-analysis. Ir J Med Sci. 2014 Jun; 183(2): 283-291. https : //doi .org/10.1 007/s11845-013-1007-x

[17] Wu VC, Chueh SJ, Chen L, et al. Risk of new-onset diabetes mellitus in primary aldosteronism: a population study over 5 years. $\mathrm{J}$ Hyperten. 2017 Aug; 35(8): 1698-1708. https://doi.org/10.1 097/HJH. 0000000000001361

[18] Funder JW, Carey RM, Mantero F, et al. The management of primary aldosteronism: case detection, diagnosis, and treatment: an endocrine society clinical practice guideline. J. Clin Endocrinol Metab. 2016 May; 101(5): 1889-1916. https://doi.org/10.1210/jc.201 5-4061

[19] Mulatero P, Stowasser M, Loh KC, et al. Increased diagnosis of primary aldosteronism, including surgically correctable forms in centers from five continents. J Clin Endorinol Metab. 2004 Mar; 89(3): 1045-1050. https://doi .org/10.1210/jc. 2003-031337

[20] Vilela LAP, Almeida MQ. Diagnosis and management of primary aldosteronism. Arch Endocrinol Metab. 2017 May-Jun; 61(3): 305-312. https://doi .org/10.1590/2359-3997000000274

[21] Dekkers T, Prejbisz A, Kool Prof L, et al. Adrenal vein sampling versus CT scan to determine treatment in primary aldosteronism: an outcome-based randomised diagnostic trial. Lancet Diabetes \& Endocrinology. 2016 Sep; 4(9): 739-746. https : //doi .org/10.1 016/S2213-8587 (16) 30100-0 